Eva Büschi, Manuela Schicka, Stefania Calabrese, Benedikt Hassler und Natalie Zambrino

\title{
Freiheitsbeschränkende Massnahmen im Umgang mit herausfordernden Verhaltensweisen von Erwachsenen mit kognitiven Beeinträchtigungen
}

Zusammenfassung: Eine Befragung von 172 Leitenden von Institutionen für Erwachsene mit kognitiven Beeinträchtigungen der Schweiz ergab, dass im Umgang mit Menschen, die herausfordernde Verhaltensweisen (HEVE) zeigen, häufig freiheitsbeschränkende Massnahmen (FBM) eingesetzt werden. Rund 80 Prozent der Leitungspersonen gaben an, dass in ihren Einrichtungen in Eskalationssituationen FBM angewendet werden. Am häufigsten werden das Separieren im privaten Zimmer, die Abgabe sedierender Medikamente, das Festhalten oder andere körperliche Interventionen sowie das Separieren in einem anderen Raum praktiziert. Auch agogische Massnahmen wie Begleitung aus dem Raum, Wechsel der Begleitperson und das Anbieten von Rückzugs-, Bewegungs- oder Entspannungsmöglichkeiten nennen die Institutionsleitenden in Bezug auf den Umgang mit HEVE.

Schlüsselwörter: Kognitive Beeinträchtigung, FBM, Sachbeschädigung, Aggression, herausfordernde Verhaltensweise, Behinderung, Gewalt, Heim.

\section{Restrictive measures in dealing with challenging behaviour of adults with cognitive disabilities}

Summary: In a Swiss study, 172 directors of institutions supporting adults with intellectual disabilities (ID) provided information about interventions used when dealing with challenging behaviours of their residents. About $80 \%$ of the directors mentioned the use of restrictive measures when supporting adults with ID during crisis intervention. Most often, they mentioned measures such as separation of clients into their private rooms, dispense of sedatives, use of fixations, physical interventions and separation into other rooms. As alternative measures, they emphasised agogic measures such as to accompany someone out of the room, to exchange the caregiver and to provide possibilities to relax, to do physical activities and/or to retreat. Keywords: Intellectual disability, restraints, restrictive measures, aggression, challenging behaviour, property damage, violence, institution, residential care institution. 


\section{Ausgangslage}

Herausfordernde Verhaltensweisen (HEVE) sind ein bedeutendes Phänomen in der aktuellen agogischen Praxis. Das Spektrum von herausfordernden Verhaltensweisen ist breit und umfasst unter anderem selbst- und fremdverletzende Verhaltensweisen sowie Sachbeschädigungen. Einerseits sind diese Verhaltensweisen ein Ausdruck von Herausforderung der Menschen mit kognitiven Beeinträchtigungen, die sie zeigen. Andererseits sind sie herausfordernd für deren Begleitpersonen, die dadurch ebenso psychischen und physischen Belastungen ausgesetzt sein können. Entsprechend stellen HEVE für Institutionen oft Schwierigkeiten dar, für die spezifische Lösungsansätze entwickelt werden müssen, um adäquat damit umzugehen.

Neben zahlreichen agogischen und therapeutischen Massnahmen werden in Institutionen des Behindertenbereichs auch freiheitsbeschränkende Massnahmen (FBM) eingesetzt. Studien zufolge sind diese «weit verbreitet in der institutionellen Praxis» (Heyvaert et al. 2014, S. 498ff.; Matson/Boisjoli 2009, S. 111). Erforscht sind FBM bisher noch kaum. In der Schweiz besteht für den Behindertenbereich weder eine nationale Erfassung der angewandten FBM noch ein Monitoring; auch Forschungsstudien hierzu fehlen bislang.

Für Pflegeheime im Altersbereich zeigten Feng et al. (2009, S. 1110) eine Prävalenz von FBM physischer Art in der Höhe von $6 \%$ auf und für den Gebrauch von Antipsychotika eine Prävalenz von $34 \%$. Klie et al. (2012) evaluierten im Alters- und Pflegebereich die ReduFix Initiative von CURAVIVA, in der die Wirkung von Qualifikationsmassnahmen auf Ausmass und Umfang von FBM geprüft und Schulungsangebote zur Sensibilisierung bezüglich FBM realisiert wurden. Schorro (2015) zeigt ebenfalls im Pflegebereich in einer Querschnittstudie zu zwei Kantonen eine Prävalenz von FBM von $26.8 \%$ auf (vgl. ebd., S. 75). Er moniert jedoch, dass weitere Daten zur Häufigkeit des Einsatzes von FBM fehlen (vgl. ebd., S. I) und fordert «spezifische Massnahmen zur Reduktion von FBM.» (ebd., S. 75) In Bezug auf Pflegeheime halten Hofmann/Schorro (2017) fest, dass die Anwendung von bewegungseinschränkenden Massnahmen «oft einhergeht mit einem unverhältnismässigen Eingriff in das Prinzip der Achtung vor der Autonomie älterer Menschen.» (ebd. S. 154)

Diskussionen über Zulässigkeit und Legitimation von FBM zeigen, wie aktuell und gleichzeitig spannungsgeladen die Thematik ist. Entsprechend soll nachfolgend auf FBM als eine spezifische Form des Umgangs 
mit herausfordernden Verhaltensweisen von Erwachsenen mit kognitiven Beeinträchtigungen genauer eingegangen werden.

\section{Rechtliche Grundlagen und Begriffsbestimmung}

Zunächst wird kurz auf die rechtlichen Grundlagen eingegangen, bevor der Begriff der FBM geklärt wird.

\section{Rechtliche Grundlagen}

FBM sind oft schwerwiegende Eingriffe in die physische und psychische Freiheit des Individuums, das von ihnen betroffen ist. Entsprechend sind sie ausreichend bestimmt zu regeln und grundsätzlich in einem formellen Gesetz zu verankern (vgl. Art. 36 BV).

Die Behindertenrechtskonvention der Vereinten Nationen (UNBRK) bezieht sich auf die Allgemeine Erklärung der Menschenrechte (AEMR) und konkretisiert sie für die spezifische Lebenssituation von Menschen mit Beeinträchtigungen. So gewährleistet sie unter anderem die Freiheit und Sicherheit der Person (Art. 14 UNBRK), die Freiheit von Folter und erniedrigender Behandlung oder Strafe (Art. 15 UNBRK), die Freiheit von Ausbeutung, Gewalt oder Missbrauch (Art. 16 UNBRK) und den Schutz der Unversehrtheit einer Person (Art. 17 UNBRK) (vgl. UNBRK).

In der Europäischen Menschenrechtskonvention (EMRK) sind insbesondere das Verbot von Folter (Art. 3 EMRK) und das Recht auf Freiheit und Sicherheit (Art. 5 EMRK) relevant. Diese halten fest, dass niemand «unmenschlicher oder erniedrigender Strafe oder Behandlung unterworfen» werden darf und regeln den rechtmässigen Freiheitsentzug (vgl. EMRK).

In der Schweizerischen Bundesverfassung (BV) sind im Zusammenhang mit FBM insbesondere zentrale Rechte wie die Rechtsgleichheit (Art. 8 BV), das Recht auf Leben und persönliche Freiheit (Art. 10 BV), der Schutz der Privatsphäre (Art. 13 BV) und das Recht auf Meinungs- und Informationsfreiheit (Art. 16 BV) zu nennen (vgl. BV).

Das Schweizerische Zivilgesetzbuch (ZGB) beinhaltet insbesondere den allgemeinen Schutz der Persönlichkeit gegen Verletzungen (Art. $28 \mathrm{ff}$. ZGB) und den Erwachsenenschutz (Art. $360 \mathrm{ff}$. ZGB). Es werden gesamtschweizerisch die Voraussetzungen festgelegt, die in Institutionen gegeben sein müssen, um Einschränkungen der Bewegungsfreiheit vorzunehmen (Art. 383 ZGB). Zudem werden deren Protokollierung und Information (Art. 384 ZGB) sowie der prozessuale Schutz der Persönlichkeit (Art. 385 ZGB) geregelt (vgl. ZGB). 
Im ZGB wird zwischen urteilsunfähigen und urteilsfähigen Personen unterschieden. Für urteilsfähige Personen in Institutionen gibt es keine explizite gesetzliche Regelung. Daher gilt der Grundsatz, dass ohne ihre Einwilligung keine bewegungseinschränkenden Massnahmen getroffen werden dürfen. Die Voraussetzungen für die Einschränkung der Bewegungsfreiheit bei einer urteilsunfähigen Person sind so geregelt, dass eine Institution deren Bewegungsfreiheit nur einschränken darf, «wenn weniger einschneidende Massnahmen nicht ausreichen oder von vornherein als ungenügend erscheinen und die Massnahme dazu dient:

, eine ernsthafte Gefahr für das Leben oder die körperliche Integrität der betroffenen Person oder Dritter abzuwenden; oder

, eine schwerwiegende Störung des Gemeinschaftslebens zu beseitigen.» (Art. 383 ZGB)

Ausserdem ist die Person über die Ausgestaltung, Dauer und Begleitperson zu informieren und die Einschränkung «so bald wie möglich wieder aufzuheben und auf jeden Fall regelmässig auf ihre Berechtigung hin» zu überprüfen (ebd.). Die Einschränkung muss stets verhältnismässig sein. Das Erwachsenenschutzrecht (in den Art. 360 bis 455 ZGB) regelt zudem die Voraussetzungen für Erwachsenenschutzmassnahmen sowie die Rechte, Pflichten und Zuständigkeiten von Mandatsführenden, welche urteilsunfähige Personen vertreten (vgl. KESG).

Zudem wird in der Botschaft zum ZGB explizit betont, dass die Mitarbeitenden angehalten sind, «die Umstände jedes Einzelfalls vertieft zu prüfen und nicht voreilig eine Massnahme anzuordnen.» (Botschaft zur Änderung des ZGB, BBI 2006, 7001) Weiter wird verdeutlicht, dass es in der Verantwortung der Institution liegt, «in einem internen Reglement festzulegen, wer eine Massnahme zur Einschränkung der Bewegungsfreiheit anordnen darf. (...) Die Dauer der Massnahme ist den Umständen des Einzelfalls anzupassen.» (ebd.).

\section{Freiheitsbeschränkende Massnahmen}

Der Begriff der Einschränkung der Bewegungsfreiheit ist gemäss Botschaft zur Änderung des ZGB weit zu verstehen und umfasst:

, elektronische Überwachungsmassnahmen

, das Abschliessen von Türen

> das Anbringen von Bettgittern und anderen Schranken

, das Angurten zur Vermeidung von Stürzen (vgl. Botschaft zur Änderung des ZGB, BBl 2006, 7001). 
Hingegen wird laut Botschaft das «Ruhigstellen durch Medikamente» nicht unter dem Begriff «Einschränkung der Bewegungsfreiheit» subsumiert, sondern fällt unter die Regelung über medizinische Massnahmen (Art. 377ff. oder 433 ff. ZGB) (vgl. Botschaft zur Änderung des ZGB, BBl 2006, 7001).

Trotz dieser unterschiedlichen Verortung in den gesetzlichen Grundlagen wurde in vorliegender Studie auch die Massnahme «Ruhigstellen durch Medikamente» unter dem Begriff «Abgabe von sedierenden Medikamenten» erhoben. Damit sind Arzneimittel mit beruhigenden Eigenschaften gemeint. Diese Massnahme wurde zusätzlich zu den oben genannten physischen bzw. räumlichen Massnahmen als eine mögliche Art der «Einschränkung der Bewegungsfreiheit» erfasst. In der Folge wird daher der übergeordnete Begriff «Freiheitsbeschränkende Massnahmen» oder «FBM» genutzt, um sowohl physische Massnahmen (wie Fixierungen, Arretieren des Rollstuhls, Festhalten und andere), wie auch räumliche Massnahmen (Separation und andere) als auch die Abgabe von sedierenden Medikamenten (medizinische Massnahme) zu berücksichtigen. Mit Anderer/ Mösch (2016) kann somit treffend formuliert werden: «Freiheitsbeschränkende Massnahmen sind alle Massnahmen, mit denen in die körperliche und geistige Unversehrtheit oder/und in die Bewegungsfreiheit eingegriffen wird, ohne dass dafür eine gültige, aktuelle und erklärte Zustimmung des Betroffenen vorliegt, bzw. ohne dass die Massnahme dem mutmasslichen Willen des kommunikationsunfähigen Betroffenen entspricht.»

Freiheitsbeschränkende Massnahmen (FBM) umfassen:

Einschränkung der Bewegungsfreiheit (ZGB 383)

> Physische Massnahmen (Fixierungen, Festhalten usw.)

, Räumliche Massnahmen (Separation, Isolation usw.)

Medizinische Massnahmen (ZGB 377ff.; $433 \mathrm{ff.)}$

, Medizinische Massnahmen (Ruhigstellen durch sedierende Medikamente)

\section{Zielsetzung und Fragestellungen}

Ziel dieses Artikels ist es, den Umgang mit FBM bei herausfordernden Verhaltensweisen von Erwachsenen mit kognitiven Beeinträchtigungen in Institutionen des Behindertenbereichs in der Schweiz aufzuzeigen. Dabei sollen folgende Fragestellungen beantwortet werden:

, Wie häufigwerden FBM im Umgang mit herausfordernden Verhaltensweisen in Institutionen des Behindertenbereichs angewendet? 
, Welche Zusammenhänge bestehen zwischen herausfordernden Verhaltensweisen und der Anwendung von FBM?

, Welche alternativen Massnahmen zu FBM werden im Umgang mit herausfordernden Verhaltensweisen angewendet?

\section{Methodisches Vorgehen}

Zunächst wird der Begriff herausfordernde Verhaltensweisen geklärt, dann die Datenerhebung und Datenauswertung erläutert, bevor im Anschluss daran die Stichprobe beschrieben wird.

Herausfordernde Verhaltensweisen werden im Rahmen dieser Studie systemökologisch betrachtet und somit als Produkt von Wechselbeziehungen zwischen den Menschen mit kognitiven Beeinträchtigungen und deren Umwelt gesehen (vgl. Calabrese 2017; Theunissen 2019a; Palmowski 2015). In Theorie und Praxis besteht ein heterogenes Verständnis von herausfordernden Verhaltensweisen (vgl. Theunissen 2016). Um sicherzustellen, dass alle Befragten von ähnlichen Grundlagen ausgehen, wurde in der durchgeführten Fragebogenerhebung folgende Definition verwendet:

\section{Herausfordernde Verhaltensweisen}

, umfassen externalisierende (z.B. fremdverletzende, selbstverletzende, gemeinschaftsstörende oder sachbeschädigende) Verhaltensweisen und/oder internalisierende Verhaltensweisen (z.B. Antriebslosigkeit oder Rückzugstendenzen);

, können sich mittels spezifischer Anzeichen ankündigen oder (scheinbar) abrupt und plötzlich auftreten;

, können gezielt ausgeübt und gerichtet wirken oder aber eher impulsiv, unkontrolliert und unberechenbar (i.S. eines Kontrollverlusts);

, zeigen sich über einen längeren Zeitraum, sind wiederholt beobachtbar und treten in einer bestimmten Häufigkeit und Intensität zutage;

, sind einerseits Ausdruck von erlebten Herausforderungen der Person selbst und fordern andererseits Begleitpersonen, Mitbewohnende, Angehörige und Institutionen heraus (vgl. Büschi et al. 2015; Wüllenweber 2009).

\section{Datenerhebung und Datenauswertung}

In einer ersten Phase des Forschungsprojekts wurden im Zeitraum von Mai bis Oktober 2018 alle erfassten Leitungspersonen von Institutionen für Menschen mit kognitiven Beeinträchtigungen in der Schweiz mittels 
eines standardisierten Online-Fragebogens zu herausfordernden Verhaltensweisen befragt. Hierbei ging es insbesondere um Vorkommen und Formen von herausfordernden Verhaltensweisen, aber auch um den Umgang damit. Dabei wurde die Anwendung von FBM erfasst. Zusätzlich wurden Angaben zu strukturellen Rahmenbedingungen erhoben.

Mangels Registerdaten, die Auskunft über die einzelnen Institutionen für Menschen mit kognitiven Beeinträchtigungen geben könnten, wurden mittels verschiedener Quellen 380 Institutionen in der Schweiz identifiziert, die Wohnplätze für erwachsene Menschen mit kognitiven Beeinträchtigungen anbieten. Die im Rahmen der Recherche verwendeten Quellen waren Verzeichnisse der großen Branchenverbände CURAVIVA und INSOS, Tabellen der Kantone, die Webseite http://www.heiminfo.ch, die Datenbank der Konferenz der kantonalen Sozialdirektorinnen und Sozialdirektoren (SODK) sowie hochschulintern verfügbare Adresslisten.

Von den eruierten 380 Institutionen befinden sich 318 in der Deutschschweiz, 52 in der Westschweiz und zehn im Tessin. Nachdem diese kontaktiert wurden, zeigte sich, dass 23 Institutionen fälschlicherweise ins Sample aufgenommen worden waren. Einige Institutionen boten ausschliesslich Plätze für Kinder und Jugendliche oder für Menschen mit psychischen Beeinträchtigungen an. Ein paar wenige Institutionen existierten nicht mehr, waren aber nach wie vor in den verfügbaren Quellen aufgeführt. Somit verblieben 357 Institutionen in der Stichprobe. Hiervon nahmen 172 Leitungspersonen an der Umfrage teil, was einer Rücklaufquote von $48.2 \%$ entspricht.

Für die Auswertung der Daten wurde die Statistiksoftware SPSS Statistics 24.0 verwendet. Die erhobenen Daten wurden dafür in das Programm importiert und zunächst bereinigt. Anschliessend erfolgten sowohl deskriptive als auch bivariate Analysen.

\section{Stichprobe}

Tabelle 1 beschreibt das Sample. Die meisten Institutionen verfügen über 31 bis 80 Wohnplätze für Menschen mit kognitiven Beeinträchtigungen. Im Durchschnitt hat eine Institution 55 Bewohnende, wobei der Median mit 35 deutlich niedriger liegt. Etwa ein Drittel der Institutionen bietet Plätze mit Intensivbetreuung an. Die grosse Mehrheit der Institutionen hat einen internen Arbeitsbereich, in dem die Bewohnenden einer Tätigkeit nachgehen können. Etwa zwei Drittel der Wohngruppen liegen in ländlichen Gegenden, wohingegen nur etwas über zehn Prozent in Städten oder Grossräumen mit mehr als 100000 Einwohnern liegen. 
Tabelle 1:

\begin{tabular}{|c|c|c|}
\hline & $\mathrm{N}$ & $\%$ \\
\hline \multicolumn{3}{|l|}{ Anzahl Bewohnende $(\mathrm{N}=171)$} \\
\hline $1-10$ & 28 & 16.4 \\
\hline $11-30$ & 49 & 28.7 \\
\hline $31-80$ & 55 & 32.2 \\
\hline$>80$ & 39 & 22.7 \\
\hline \multicolumn{3}{|l|}{ Plätze mit Intensivbetreuung $(\mathrm{N}=172)$} \\
\hline Ja & 67 & 39.0 \\
\hline Nein & 105 & 61.0 \\
\hline \multicolumn{3}{|l|}{ Interner Arbeitsbereich $(\mathrm{N}=172)$} \\
\hline $\mathrm{Ja}$ & 148 & 86.0 \\
\hline Nein & 24 & 14.0 \\
\hline \multicolumn{3}{|l|}{ Anzahl Wohngruppen $(\mathrm{N}=172)$} \\
\hline Keine Wohngruppen & 8 & $4 \cdot 7$ \\
\hline $1-3$ & 50 & 29.1 \\
\hline $4-10$ & 78 & $45 \cdot 3$ \\
\hline$>10$ & 36 & 20.9 \\
\hline \multicolumn{3}{|l|}{ Lage der Wohngruppen $\left(\mathrm{N}=199^{\star}\right)$} \\
\hline Ländliche Gegend & 115 & 66.9 \\
\hline Stadt $<20000$ Einwohner & 42 & 24.4 \\
\hline Stadt 20000-100 o00 Einwohner & 24 & 14.0 \\
\hline Stadt/Grossraum > 100000 Einwohner & 18 & 10.5 \\
\hline
\end{tabular}

* Mehrfachnennungen möglich

Die Institutionsleitenden wurden gebeten, eine Selbsteinschätzung vorzunehmen und auf einer endpunktbenannten Skala von 1 bis 5 zu beurteilen, ob ihre Institution auf den Umgang mit HEVE spezialisiert ist ( $1=$ nicht spezialisiert; $5=$ hoch spezialisiert). Knapp $35 \%$ der Befragten schätzen ihre Institution als spezialisiert oder hoch spezialisiert ein (Werte 4 und 5), wohingegen knapp $31 \%$ diese als nicht oder wenig spezialisiert bezeichnen (Werte 1 und 2). Die restlichen 34\% der Institutsleitenden geben eine neutrale Einschätzung (Wert 3) bezüglich der HEVE-Spezialisierung ihrer Institution an. Auf HEVE spezialisierte Institutionen verfügen eher über Plätze mit Intensivbetreuung und Zusatzfinanzierung und nutzen häufiger Sonderplätze als nicht spezialisierte Institutionen (vgl. Calabrese et al. 2019). Die HEVE-Prävalenz (Anzahl der Bewohnenden mit HEVE/Anzahl der Bewohnenden insgesamt) liegt bei $28.2 \%$, und ist etwas höher bei Männern (31.1\%) als bei Frauen (24.7\%). 


\section{Ergebnisse}

Nachfolgend werden zentrale Ergebnisse zu FBM aus der Sicht der Institutionsleitenden präsentiert. Wie erwähnt, wurden die FBM im Fragebogen unterteilt in: Physische Massnahmen; räumliche Massnahmen und die Abgabe sedierender Medikamente.

Zunächst wird auf die Häufigkeit und Anwendung von FBM fokussiert. Im Anschluss daran wird auf FBM im Umgang mit HEVE eingegangen, wobei der Blick auf allgemeine Zusammenhänge zwischen HEVE und FBM gerichtet wird. Abschliessend werden Alternativen zu FBM dargelegt.

\section{Häufigkeit und Anwendung von FBM als präventive Massnahmen}

Die Institutionsleitenden wurden gebeten, anzugeben, welche präventiven Massnahmen in den von ihnen geleiteten Institutionen im Umgang mit Menschen mit HEVE zur Anwendung kommen. Abbildung 1 zeigt, dass sie vor allem Kommunikation (inkl. Hilfsmittel), regelmässige Einzelaktivitäten im 1:1 Setting, Aktivitäten in der Natur und Bewegungsoder Wasserangebote erwähnten. Auch musikalische Angebote, TEACCH, Entspannungsangebote, Psychotherapie, Massagen, verhaltenstherapeutische Massnahmen und Videoanalyse wurden genannt. Unter offenen Angaben zu präventiven Massnahmen wurden zudem Sportangebote, kreative, gestalterische und handwerkliche Angebote, lebenspraktische Tätigkeiten, anthroposophische und therapeutische Angebote (insbesondere körperbezogene und tiergestützte Therapie) aufgeführt. Auch Rollenspiele, Coaching und Schulung der Personen, die HEVE zeigen, zu bestimmten Themen (wie Prävention, Sexualität, Verhaltenskodex, Aggressionsmanagement) sowie spezifische, individuell angepasste Einzelangebote wurden genannt.

Abbildung 1 zeigt darüber hinaus, dass nach Angaben der Institutionsleitenden neben den genannten vielfältigen agogischen Angeboten auch medizinische Massnahmen und FBM zur Prävention eingesetzt werden. Medizinische Präventionsmassnahmen umfassen Medikation und somatische Versorgung. Als FBM eingestuft werden Hilfsmittel zur Verhinderung von Selbstverletzungen (30.0\%) ebenso wie Fixiervorrichtungen (29.1\%), Gurtsysteme und andere physische Massnahmen (27.3\%).

\section{Häufigkeit und Anwendung von FBM in Eskalationssituationen}

Weiter wurden die Institutionsleitenden gefragt, welche Massnahmen in ihren Institutionen in Eskalationssituationen angewendet werden. Darunter werden Situationen verstanden, in denen HEVE gezeigt werden und akuter Handlungsbedarf besteht, um die Sicherheit aller zu gewährleisten. Die 
Abbildung 1

Agogische, medizinische und freiheitsbeschränkende

Präventionsmassnahmen

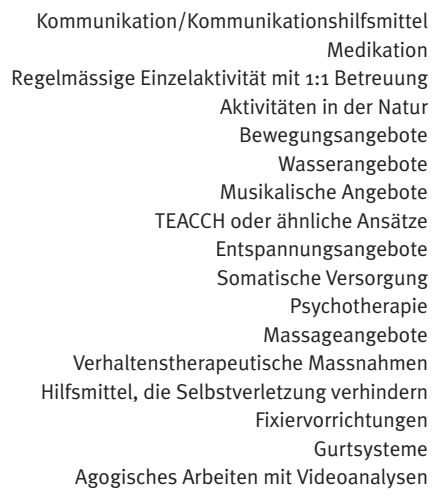

.

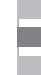

西

\section{.}

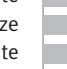

.

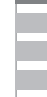

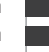

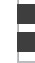

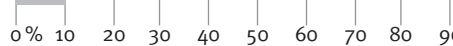

$\mathrm{N}=172$; Mehrfachnennungen möglich.

Institutionsleitenden erwähnten als agogische Massnahmen in Eskalationssituationen die Begleitung aus dem Raum, das Anbieten von Bewegungsmöglichkeiten, die Aufrechterhaltung des Kontakts, den Wechsel der Begleitperson, die Ablenkung, das gemeinsame Aushalten der Situation sowie die Veränderung der räumlichen Atmosphäre. Auch FBM werden in Eskalationssituationen angewendet. Diese unterscheiden sich jedoch von den FBM, die zur Prävention von HEVE eingesetzt wurden, wie Abbildung 2 zeigt.

FBM werden in Eskalationssituationen oft angewendet. So gaben 80.2\% der Institutionsleitenden an, dass in ihren Institutionen bei Eskalationen FBM zum Einsatz kommen (siehe Tabelle 2). Die am häufigsten angewendeten Massnahmen sind das Separieren im privaten Zimmer (63.4 \%), die Abgabe sedierender Medikamente (54.1\%), das Festhalten oder andere körperliche Interventionen (40.7\%) und das Separieren in einem anderen Raum (38.4\%) (vgl. Abbildung 2). Fixierungen werden deutlich seltener vorgenommen. Von den teilnehmenden Institutionsleitenden gab niemand an, 7-Punkt-Fixierungen oder Zwangsjacken anzuwenden.

Die Institutionsleitenden, in deren Institutionen FBM angewendet werden, wurden gefragt, in wie vielen Eskalationssituationen gemäss ihrer Schätzung FBM angewendet werden. Dabei konnten sie auswählen zwischen

a) in $0-25 \%$ der Situationen,

b) in $26-50 \%$ der Situationen, 


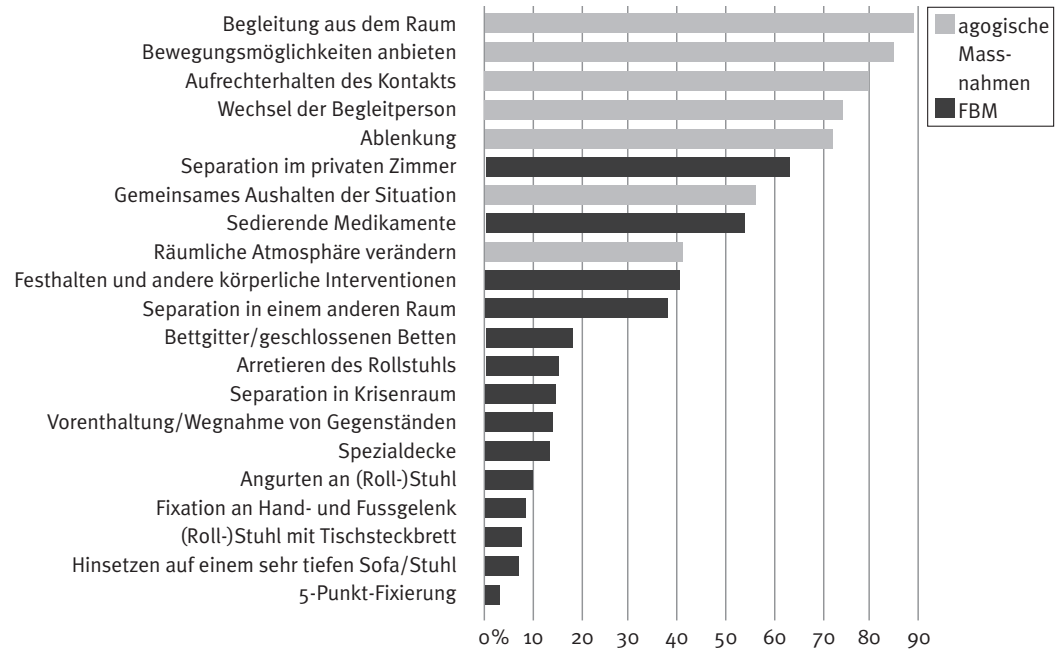

$\mathrm{N}=172$; Mehrfachnennungen möglich.

c) in $51-75 \%$ der Situationen und

d) in 76-100\% der Situationen.

Von den Institutionen, in denen FBM zum Einsatz kommen, geschieht dies zu $72.5 \%$ in einer von vier Eskalationssituationen. Bei 27.5\% kommen FBM häufiger als in jeder vierten Eskalationssituation zur Anwendung.

In $84.8 \%$ der Institutionen, in denen FBM angewendet werden, ist ein Konzept zur Anwendung von FBM vorhanden. In 92.8\% der Institutionen werden die Anwendungen von FBM wie gesetzlich vorgegeben protokolliert (vgl. Art. 384 ZGB). Auch erfolgt in den allermeisten Institutionen (93.5\%) eine entsprechende Schulung der Mitarbeitenden zu FBM.

Bei $6.5 \%$ der Institutionen erfolgt keine Schulung der Mitarbeitenden bezüglich FBM. Es wird zudem ersichtlich, dass $15.2 \%$ der Institutionen über kein Konzept zur Anwendung von FBM verfügen und in $7.2 \%$ der Institutionen entgegen den gesetzlichen Vorgaben angewandte FBM nicht protokolliert werden.

Für die Anwendung von FBM in Eskalationssituationen ist der Grad, in dem Institutionen auf HEVE spezialisiert sind, von Bedeutung. Tabelle 2 zeigt, dass in 35 der 53 Institutionen, die sich selbst als nicht oder nur gering auf HEVE spezialisiert beschreiben, FBM zur Anwendung kommen. Bei den auf HEVE spezialisierten Institutionen kommen FBM bei 55 
von 60 Institutionen zur Anwendung. Der Zusammenhang zwischen dem HEVE-Spezialisierungsgrad und der Anwendung von FBM ist statistisch signifikant (Cramérs V=0.261, p=0.003).

\begin{tabular}{|c|c|c|c|c|}
\hline \multirow{2}{*}{\multicolumn{2}{|c|}{ Spezialisierungsgrad der Institution }} & \multicolumn{3}{|c|}{ Anwendung FBM } \\
\hline & & Nein & $\mathrm{Ja}$ & Gesamt \\
\hline nicht/ge & alisiert & 18 & 35 & 53 \\
\hline mittel sp & & 11 & 48 & 59 \\
\hline spezialis & spezialisiert & 5 & 55 & 60 \\
\hline \multirow[t]{2}{*}{ Gesamt } & $\mathrm{N}$ & 34 & 138 & 172 \\
\hline & $\%$ & 19.8 & 80.2 & 100.0 \\
\hline
\end{tabular}

\begin{tabular}{|c|c|c|c|c|}
\hline \multirow{2}{*}{\multicolumn{2}{|c|}{ Anzahl Bewohnende }} & \multicolumn{3}{|c|}{ Anwendung FBM } \\
\hline & & Nein & $\mathrm{Ja}$ & Gesamt \\
\hline $01-10$ & & 13 & 15 & 28 \\
\hline $11-30$ & & 11 & 38 & 49 \\
\hline $31-80$ & & 6 & 49 & 55 \\
\hline \multirow[t]{2}{*}{ Gesamt } & $\mathrm{N}$ & 33 & 138 & 171 \\
\hline & $\%$ & $19 \cdot 3$ & 80.7 & 100.0 \\
\hline
\end{tabular}

Auch die Anzahl der Bewohnenden steht in einem signifikanten Zusammenhang dazu, ob FBM in Eskalationssituationen angewendet werden oder nicht (Cramérs $\mathrm{V}=0.337 ; \mathrm{p}=0.000$ ). Von den 28 kleinen Institutionen im Sample (Anzahl Bewohnende unter elf) geben 13 an, keine FBM in Eskalationssituationen anzuwenden. Von den 39 grossen Institutionen (Anzahl Bewohnende von mehr als 80) verzichten hingegen lediglich drei Institutionen vollständig auf die Anwendung von FBM (siehe Tabelle 3).

Die Anwendung von FBM in Eskalationssituationen hängt auch mit der Belastung der Mitarbeitenden zusammen. So beantworteten 68 der 138 Institutionsleitenden von Institutionen, in denen FBM zur Anwendung kommen, die Frage, ob es in der Institution Begleitpersonen gibt, die nach herausfordernden Verhaltensweisen von Klientinnen und Klienten arbeitsunfähig waren oder sind mit «Ja». Bei den Institutionen, in denen keine FBM zur Anwendung kommen sind dies lediglich 4 von 34 Instituti- 
Tabelle 4:

Arbeitsunfähigkeit der Mitarbeitenden in Abhängigkeit von der Anwendung von FBM in Institutionen $(N=172)$

Arbeitsunfähigkeit Mitarbeitende Anwendung FBM

\begin{tabular}{lccr} 
& Nein & Ja & Gesamt \\
\hline Ja & 4 & 68 & 72 \\
Nein & 30 & 70 & 100
\end{tabular}

onen. Dieser Zusammenhang ist statistisch signifikant (Cramérs V=0.303; $\mathrm{p}=0.000$ ).

Tabelle 5 verdeutlicht, inwiefern der Anteil der Personen, die HEVE in einer Institution zeigen, mit der Anwendung von FBM in Eskalationssituationen zusammenhängt. Sieben von zwölf Institutionen, die keine Bewohnenden aufweisen, die HEVE zeigen, verzichten vollständig auf die Anwendung von FBM. Demgegenüber ist der Anteil der Institutionen, in denen FBM zur Anwendung kommen, unter Institutionen mit Bewohnenden, die HEVE zeigen, deutlich höher. Insgesamt ist der Zusammenhang zwischen der Anwendung von FBM und der HEVE-Prävalenz statistisch signifikant (Cramérs V=0.306, $\mathrm{p}=0.007$ ).

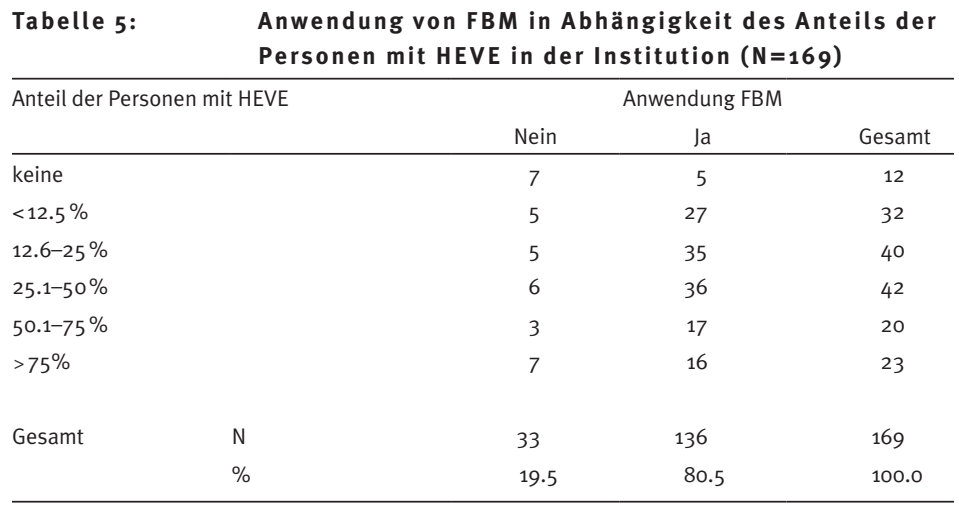

\section{Alternativen zu FBM in Eskalationssituationen}

Wie bereits dargestellt, kommen in $80.2 \%$ der teilnehmenden Institutionen FBM in Eskalationssituationen zum Einsatz. Demgemäss geben 19.8 \% der Institutionsleitenden an, in ihren Institutionen auch in Eskalationssituationen ganz auf die Anwendung von FBM zu verzichten. Dies betrifft 34 Insti- 
tutionen. In sieben dieser 34 Institutionen leben keine Menschen, die HEVE zeigen. In den anderen 27 Institutionen, die HEVE-Vorkommen verzeichnen und auf die Anwendung von FBM verzichten, kommen als Alternativen verschiedene agogische Massnahmen zum Einsatz. Genannt wird beispielsweise die Begleitung aus dem Raum, das Aufrechterhalten des Kontakts und das Anbieten von Bewegungsmöglichkeiten. Weiter wird das Anbieten von Rückzugsoptionen oder Entspannungsmöglichkeiten sowie Ablenkung oder eine räumliche Veränderung der Atmosphäre erwähnt. Zusätzliche Massnahmen (laut den offenen Angaben im Fragebogen) sind paradoxe Interventionen, der Rückzug der Begleitpersonen und der anderen Bewohnenden oder auch die lösungsorientierte Gesprächsführung (Abb. 2).

\section{Diskussion}

Nachfolgend werden die wesentlichen Ergebnisse zu FBM des Projekts in einigen Punkten diskutiert.

Nach Angaben der Institutionsleitenden werden als präventive Massnahmen neben vielfältigen agogischen Angeboten auch medizinische Massnahmen und FBM eingesetzt. Als FBM werden Hilfsmittel zur Verhinderung von Selbstverletzungen ebenso genannt wie Fixiervorrichtungen, Gurtsysteme und andere physische Massnahmen. Dieser Einsatz von FBM als Präventionsmassnahme widerspricht den gesetzlichen Grundlagen, wonach FBM «gesetzlich vorgesehen, durch ein öffentliches Interesse gerechtfertigt und verhältnismässig sein» müssen und zudem «nur als ultima ratio Massnahmen in Fällen von Selbst- oder Fremdgefährdung eingesetzt werden» sollen (vgl. NKVF 2016, S. 35). Laut NKFV ist jeweils «das mildeste Mittel und damit die am wenigsten einschneidende Massnahme zu wählen und die Massnahmen (sind) so rasch als möglich wieder aufzuheben» (ebd.). Angesichts dieser Tatsache, bedürfen FBM, die zu Präventionszwecken eingesetzt werden eines besonderen Legitimationsaufwands (vgl. Calabrese et al. 2019) und es zeigt sich, dass die Frage nach alternativen Präventionsmassnahmen zu FBM von besonderem Interesse ist.

$80.2 \%$ der befragten Institutionsleitenden gaben an, in ihrer Institution kämen in Eskalationssituationen FBM zur Anwendung. Von den Institutionen, die FBM nutzen, geschieht dies zu etwa $70 \%$ in einer von vier Eskalationssituationen. Bei knapp 30\% kommen FBM häufiger als in jeder vierten derartigen Situation zum Einsatz. Dabei bestehen die häufigsten Massnahmen im Separieren in eigenen oder anderen Räumen, in der Abgabe von sedierenden Medikamenten oder in körperlichen Massnahmen. Dies bestätigt Studien, wonach FBM generell oft eingesetzt werden 
(vgl. Matson/Boisjoli 2009); wonach die Abgabe von Medikamenten häufig erfolgt (vgl. Deb et al. 2015; Allen 2000 und für Pflegeheime in der Schweiz: Zúñiga et al. 2013) und wonach körperliche Massnahmen häufig eingesetzt werden (vgl. Matson/Boisjoli 2009).

Die Anwendung von FBM erfolgt gemäss Einschätzungen der Institutionsleitenden grösstenteils korrekt entlang der rechtlichen Vorgaben. Knapp 85\% der Befragten geben an, dass in ihrer Institution ein Konzept zur Anwendung von FBM besteht und die Anwendungen wie gesetzlich vorgegeben, protokolliert werden. Auch bieten die allermeisten Institutionen (93.5\%) ihren Mitarbeitenden eine Schulung zu FBM an. Bei einigen (wenigen) Institutionen erfolgt jedoch der Einsatz von FBM, ohne dass ein entsprechendes Konzept existiert; wird die Anwendung von FBM (entgegen der gesetzlichen Vorgabe) nicht protokolliert und werden FBM umgesetzt, ohne dass Mitarbeitende diesbezüglich geschult werden. Da jede FBM einen Eingriff in die Grundrechte der davon betroffenen Menschen darstellt, ist es zentral, diese Massnahmen zu legitimieren. Es gilt die rechtlichen Grundlagen einzuhalten und die Güterabwägungen zwischen Freiheit und Schutz sorgfältig vorzunehmen (vgl. Mösch 2018, S. 75). Entsprechend sind fehlende Konzepte und Protokolle im Zusammenhang mit FBM als gravierende Fehler zu bezeichnen, die Institutionsleitende vermeiden sollten. FBM sind wie erwähnt nur als ultima ratio Massnahmen anzuwenden und insgesamt muss der agogische Anspruch sein, beim Umgang mit HEVE auf FBM zu verzichten. Ist dies - in extremen Situationen mit massiven HEVE - nicht möglich, gilt es, die FBM gemäss den rechtlichen Grundlagen anzuwenden, regelmässig kritisch zu überprüfen und mithilfe der Entwicklung agogischer Massnahmen zu reduzieren. Wesentlich für die Reduktion von FBM ist gemäss Evaluation der ReduFix Initiative von CURAVIVA (bezogen auf Pflegeheime), dass ein Bündel an Massnahmen getroffen wird, zu denen unter anderen die Erstellung von hausinternen Konzepten und die Weiterbildung der Mitarbeitenden hinsichtlich FBM gehören (vgl. Klie et al. 2012). Nur so können ethische Prinzipien wie die Wahrung von Würde, Selbstbestimmung, Autonomie und persönlicher Integrität adäquat berücksichtigt werden (vgl. Hofmann/Schorro 2017: 151). Entsprechend ist bei Institutionsleitenden das Bewusstsein für die Diffizilität von FBM zu schärfen und sind auch die Begleitpersonen, die FBM anwenden, dafür zu sensibilisieren. Guidelines für die Güterabwägungen wie sie in der Medizin oder Alterspflege bestehen (vgl. Mösch 2018, S. 67f.), wären auch für den Behindertenbereich wichtig und sind noch zu entwickeln. Es darf nicht sein, dass mittels FBM Eingriffe in die Grundrechte leichtfertig vorge- 
nommen werden. Alternativen zu FBM sind systematisch zu prüfen und bei Unsicherheiten bezüglich der Voraussetzungen und Grenzen der Anwendung von FBM sollte eine Kombination aus (externer) juristischer und agogischer Beratung zur Verfügung stehen und genutzt werden.

Relevante Faktoren für die Anwendung von FBM in Eskalationssituationen sind der Spezialisierungsgrad und die Anzahl der Bewohnenden einer Institution: Nicht auf HEVE spezialisierte Institutionen verzichten eher ganz auf den Einsatz von FBM, als auf HEVE spezialisierte Institutionen. Eine Erklärung dafür könnte sein, dass nicht spezialisierte Institutionen weniger Personen aufweisen, die HEVE zeigen. Dementsprechend kann es sein, dass spezialisierte Institutionen (gerade aufgrund ihrer Spezialisierung) eher viele Personen mit HEVE aufweisen, über ein Sondersetting verfügen und damit das Risiko besteht, dass auf HEVE in (alltäglichen) Eskalationssituationen - entgegen dem ultima ratio Grundsatz - relativ zügig mit FBM reagiert wird, ähnlich wie dies aus Sondersettings bekannt ist (vgl. Theunissen 2019b). Glasenapp (2013, S. 97) beschreibt dies als «Schwächung ethischer Standards», die durch wechselseitige Verstärkung von dysfunktionalen Verarbeitungsprozessen aufgrund des alltäglichen Erlebens von herausfordernden Verhaltensweisen ausgelöst wird. Auch die Anzahl der Bewohnenden steht in einem signifikanten Zusammenhang damit, ob FBM in Eskalationssituationen angewendet werden oder nicht. Von den kleinen Institutionen im Sample (Anzahl Bewohnende unter elf) geben einige an, in Eskalationssituationen keine FBM anzuwenden. Von den grossen Institutionen (mit einer Anzahl Bewohnenden von mehr als 80) verzichtet hingegen nur ein geringer Anteil auf die Anwendung von FBM. Dies könnte ein Hinweis darauf sein, dass kleinere Settings, mit einer geringen Anzahl Bewohnenden, es eher erlauben, explizit auf die Anwendung von FBM zu verzichten und im Umgang mit HEVE alternative Massnahmen zu entwickeln. Kleinere Settings fordert denn auch Theunissen, wenn er neben Formen eines unterstützten Wohnens (allein oder zu zweit) künftig nur noch Wohngruppen mit maximal vier Personen propagiert (Theunissen 2019a).

Institutionen, die auf FBM verzichten, machen knapp $20 \%$ aus. Darin enthalten sind auch einige Institutionen, in denen keine Personen mit HEVE leben. Der Verzicht auf FBM könnte also damit zusammenhängen, dass kein HEVE-Vorkommen vorliegt. Gleichzeitig verzichten 27 der teilnehmenden 172 Institutionen, die HEVE-Vorkommen aufweisen, explizit auf den Einsatz von FBM. Eine Erklärung dafür könnte eine generell kritische Haltung gegenüber FBM sein oder der Verzicht auf das Eingehen rechtlicher Risiken, die beim Einsatz von FBM oft bestehen. Mösch (2018, 
S. 75) hält diesbezüglich fest, dass FBM in Institutionen einer Rechtfertigung bedürfen und dabei oft «eine Güterabwägung zwischen Freiheit und Schutzinteressen notwendig» ist. "Heime, Einrichtungen, Pflegepersonen und die Betreuenden bewegen sich insoweit auf einem rechtlichen Feld, welches heikle Güterabwägungen notwendig macht.» (Mösch 2018, S. 75). Institutionen, die explizit auf FBM verzichten, können diese rechtlichen Risiken minimieren, indem sie statt FBM verschiedene agogische Massnahmen einsetzen. Laut Olivier-Pijpers et al. (2018, S. 250) geht es darum, in Institutionen klare und authentische Haltungen und Leitlinien zu entwickeln und den Begleitpersonen kongruentes Coaching seitens der Leitungspersonen zu bieten, um sie im agogischen Umgang mit HEVE anzuleiten. Dies setzt allerdings die Bereitstellung entsprechender Ressourcen (für Coaching, interne und externe Weiterbildung) voraus.

Als Alternativen zu FBM werden folgende agogischen Massnahmen genannt: Aus dem Raum begleiten; Aufrechterhalten des Kontakts, Anbieten von Bewegungsmöglichkeiten; Wechsel der Begleitperson und Veränderung der räumlichen Atmosphäre. Weiter wurde das Anbieten von Rückzugsoptionen oder Entspannungsmöglichkeiten genannt sowie Ablenkung. Zusätzliche Massnahmen waren paradoxe Interventionen, der Rückzug der Begleitpersonen und der anderen Bewohnenden oder auch die lösungsorientierte Gesprächsführung. Diese Alternativen zielen alle auf die Anpassung der agogischen Angebote und eine bewusste Gestaltung bzw. Veränderung der Umgebung hin und können damit einer systemökologischen Perspektive zugeordnet werden. Insbesondere für die Wirksamkeit von körperlicher Bewegung finden sich auch bei Ogg-Groenendaal et al. (2014) und Fath (2005) Hinweise.

\section{Limitationen}

In dieser Studie wurden Daten von Schweizer Institutionen, die Wohnplätze für Erwachsene mit kognitiven Beeinträchtigungen anbieten, erhoben. Da die Grundgesamtheit und deren Charakteristika nicht bekannt sind, können zur Repräsentativität der hier vorgestellten Ergebnisse keine Angaben erfolgen. Allerdings nahmen an der Befragung Institutionen aus allen Sprachregionen teil. Zudem unterscheiden sie sich in ihrer Grösse und hinsichtlich des Spezialisierungsgrads. Daher kann davon ausgegangen werden, dass ein gutes Abbild der Schweizer Institutionen im Behindertenbereich gezeigt wird. Eine Limitation kann jedoch darin bestehen, dass es sich um Einschätzungen von Institutionsleitenden handelt, die selber zu nah oder zu entfernt von der ausführenden Praxis stehen, was die 
Resultate verzerren kann. Zudem kann es sich um eine Auswahl an Institutionsleitenden handeln, die besonders an der Thematik interessiert ist, und/oder eher Institutionen mit einer höheren Prävalenz an Klientel mit HEVE leiten. Auch dies könnte zu einer Verzerrung der Resultate führen. Ebenfalls ist nicht auszuschliessen, dass gewisse Antworten trotz des gewährleisteten Datenschutzes und der Zusicherung von Anonymität im Sinne einer sozialen Erwünschtheit beeinflusst wurden. Schliesslich beruhen die Ergebnisse auf bivariaten Analysen. Diese lassen keine Aufschlüsse auf die Richtung der bestehenden Zusammenhänge zu. Zudem gilt es zu sagen, dass beobachtete bivariate Zusammenhänge durch nicht beobachtete Drittvariabeln beeinflusst werden können. Nichtsdestotrotz bieten die Ergebnisse erstmals umfassende Angaben zu FBM im Umgang mit HEVE von Erwachsenen mit kognitiven Beeinträchtigungen in Institutionen des Behindertenbereichs. Damit werden grundlegende Daten geliefert, die bislang für die Schweiz fehlten.

Fazit

Die Perspektive der Institutionsleitenden zeigt, dass FBM gerade in Eskalationssituationen häufig genutzt werden. FBM sind jedoch personenzentriert und können jene, deren Freiheit beschränkt wird, schmerzen und sie emotional belasten (vgl. MacDonald et al. 2011, S. $45 \mathrm{ff}$. und spezifisch für Krankenhäuser Köbke/Brase 2017, S. 53 ff.). Aus der Sicht von Menschen mit kognitiven Beeinträchtigungen sind restriktive Massnahmen oft kaum merklich von Missbrauch zu unterscheiden und erscheinen ihnen häufig als ungerechtfertigt (vgl. MacDonald et al. 2011: 45 ff.). Studien zum Einsatz von FBM im Kontext von Pflegeheimen in der Schweiz zeigen auf, dass diese Massnahmen für Betroffene negative Folgen haben können: So führen sie zu einer Verringerung von kognitiven Kompetenzen und Alltagsaktivitäten, zu einer höheren Abhängigkeit bezüglich des Gehens, zu Stürzen, Druckstellen und Inkontinenz (vgl. Hofmann/Schorro 2017). FBM beeinträchtigen aber auch die Lebensqualität, verschlechtern den psychischen und körperlichen Zustand, führen zu einer Abnahme von physischen Aktivitäten und einer Zunahme von herausfordernden Verhaltensweisen (vgl. ebd. und Schorro 2015: 3).

Entsprechend kommt agogischen Alternativen zu FBMim Umgang mit herausfordernden Verhaltensweisen eine grosse Bedeutung zu. Diese sind eher umweltbezogen denn personenzentriert und ermöglichen somit eine Fokussierung auf Setting und Situation sowie auf adäquate Angebote seitens der Begleitpersonen. 
Generell ist die Guideline des National Institute für Health and Care Excellence (NICE Guideline 2015) zu beachten, die besagt, dass im Umgang mit herausfordernden Verhaltensweisen jeweils die am wenigsten restriktive, aber trotzdem effektive Methode als erstes genutzt werden soll. Bei der Anwendung von FBM sind fundierte, stete, fallspezifische Reflexionen und Evaluationen zwingend, idealerweise auch unter Beizug von externen Fachpersonen aus unterschiedlichen Disziplinen (Recht, Sonderpädagogik, Soziale Arbeit, Psychologie usw.). Analog zum Alters- und Pflegebereich und zu psychiatrischen Einrichtungen wird auch für Institutionen im Behindertenbereich empfohlen, sich an den international bereits etablierten Guidelines $\mathrm{zu}$ orientieren und in Kooperation mit den Mitarbeitenden interne Richtlinien zur Anwendung von FBM zu erarbeiten und zentrale Grundsätze festzulegen, um den Einsatz von FBM kritisch zu hinterfragen, auf ein absolutes Minimum zu begrenzen und klar zu regeln (vgl. NKVF 2016).

\section{Informationen zum Forschungsprojekt}

Das Forschungsprojekt «Herausfordernde Verhaltensweisen von Erwachsenen mit kognitiven Beeinträchtigungen in Schweizer Institutionen des Behindertenbereichs» wurde vom Schweizerischen Nationalfonds (SNF) finanziert. Es war ein Kooperationsprojekt der Hochschule für Soziale Arbeit FHNW und der Hochschule Luzern - Soziale Arbeit und wurde von 2018 bis 2020 realisiert. Das Projekt rückte das Phänomen der herausfordernden Verhaltensweisen in den Vordergrund. Fragen zur Prävalenz und zur Entstehung von herausfordernden Verhaltensweisen wurden ebenso aufgegriffen, wie Fragen nach dem Umgang damit und nach deren Folgen. Das multiperspektivisch angelegte Projekt beinhaltete fünf Phasen. In zwei quantitativen Online-Befragungen wurden die Perspektiven der Institutionsleitenden (Phase 1) und Begleitpersonen (Phase 2) erfasst, während in qualitativen Erhebungen die Sichtweisen der Klientel (Phase 3), ihrer Angehörigen und weiterer in die Begleitung involvierten Personen (Phase 4) erhoben wurden. Das Ziel bestand darin, zentrale Datengrundlagen zu gewinnen und ein systemökologisches Good-Practice Modell zu entwickeln, das Fachpersonen in der Praxis Handlungsorientierungen im agogisch adäquaten Umgang mit HEVE bietet (Phase 5). www.heve.ch.

Literatur

Allen, David (2000). Recent research on physical aggression in persons with intellectual disability: an overview. In: Jour- nal of Intellectual \& Developmental

Disability, 25(1), S. 41-57.

Anderer, Karin \& Mösch, Payot Peter (2016). Aufenthalt in Wohn- oder Pflegeeinrich- 
tungen. In: Christiana Foutoulakis, Kurt Affolter-Fringeli, Yvo Biderbost, Daniel Steck (Hrsg.), Fachhandbuch Kindesund Erwachsenenschutzrecht, Zürich: Schulthess, S. 158-172.

Botschaft zur Änderung des Schweizerischen Zivilgesetzbuches (2006) https://www.admin.ch/opc/de/ federal-gazette/2006/7001.pdf [26.04.2019].

Büschi, Eva; Antener, Gabriela; Calabrese, Stefania; Kasper, Daniel; von Fellenberg, Monika. (2015). Schlussbericht zum Projekt HEVE Forschungsprojekt zu Erwachsenen mit schweren und/oder mehrfachen Beeinträchtigungen und herausfordernden Verhaltensweisen (HEVE) im Bereich Wohnen. Olten: Institut Integration und Partizipation Hochschule für Soziale Arbeit FHNW.

BV Bundesverfassung https://www. admin.ch/opc/de/classifiedcompilation/19995395/index.html [23.01.2020].

Calabrese, Stefania (2017). Herausfordernde Verhaltensweisen. Herausfordernde Situationen: Ein Perspektivenwechsel. Bad Heilbrunn: Verlag Julius Klinkhardt.

Calabrese, Stefania; Hassler, Benedikt; Büschi, Eva; Lustenberger, Natalie; Schicka, Manuela. (2019). Merkmale spezialisierter Institutionen für Menschen mit kognitiven Beeinträchtigungen und herausfordernden Verhaltensweisen. Ergebnisse der SNF-Studie HEVE. In: VHN Plus, 88, S. 1-15. https://www. reinhardt-journals.de/index.php/vhn/ article/view/152026 [23.01.2020].

Deb, Saumitra; Unwin, Gemma; Deb, Tanya (2015). Characteristics and the trajectory of psychotropic medication use in general and antipsychotics in particular among adults with an intellectual disability who exhibit aggressive behaviour. In: Journal of Intellectual Disabilitiy Research, 59(1), S. 11-25.

EMRK Europäische Menschenrechtskonvention https://www.admin.ch/opc/de/ classified-compilation/19500267/index. html [23.01.2020].
Fath, Kirsten (2005). Verhaltensauffälligkeiten und Bewegungstherapie bei Menschen mit sehr schweren Behinderungen. Theoretische Grundlagen, Praxiskonzepte und Evaluation. Marburg: Lebenshilfe-Verlag.

Feng, Zhanlian; Hirdes, John P.; Smith, Trevor Frise; Finne-Soveri, Harriet; Chi, Iris; Du Pasquier, Jean-Noel; Gilgen, Ruedi; Ikegami, Naoki; Mor, Vincent (2009). Use of physical restraints and antipsychiotic medications in nursing homes. A cross-national study. In: International Journal of Geriatric Psychiatry, 24(10), S. 1110-1118.

Glasenapp, Jan (2013). Wirkung und Nebenwirkung von Intensivbetreuung - ein Beipackzettel. In: Jan Glasenapp \& Klaus Hennike (Hrsg.), Intensivbetreuung in der Diskussion. Orientierungspunkte für Diagnostik und Therapie. Berlin: Deutsche Gesellschaft für seelische Gesundheit bei Menschen mit geistiger Behinderung e.V., S. 95-98.

Heyvaert, Mieke; Saenen, Lore; Maes, Bea; Onghena, Patrick (2014). Systematic review of restraint interventions for challenging behaviour among persons with intellectual disabilities: Focus on effectiveness in single-case experiments. In: Journal of Applied Research in Intellectual Disabilities, 27(6), S. 493-510.

Hofmann, Hedi \& Schorro, Ewald (2017). Bewegungseinschränkende Massnahmen: ein Dilemma für die Pflege in Langzeitinstitutionen. In: PflegerechtPflege in Politik, Wissenschaft und Ökonomie, 3(17), S. 147-157.

KESG Kindes- und Erwachsenenschutzgesetz http://kesg.ch/ [06.05.2020].

Klie, Thomas; Hollfelder, Thomas; Schuhmacher, Birgit (2012). Evaluation der CURAVIVA Fachbereich Alter. AGP Sozialforschung. Freiburg: Institut für angewandte Sozialforschung an der Evangelischen Hochschule Freiburg.

Köbke, Christian \& Brase, Sabine (2017). Freiheitsentziehende Maßnahmen wirksam reduzieren. In: Pflegezeitschrift, 11(70), S. 53-56. 
MacDonald, Anne; McGill, Peter; Deveau, Roy (2011). "You squeal and squeal but they just hold you down". Restrictive physical interventions and people with intellectual disabilities: service user views. In: International Journal of Positive Behavioural Support, 1(1), S. 45-52.

Matson, Johnny Lee; Boisjoli, Jessica Ann (2009). Restraint procedures and challenging behaviours in intellectual disability: an analysis of causative factors. In: Journal of Applied Research in Intellectual Disabilities, 22(2), S. 111-117.

Mösch Payot, Peter (2018). Freiheitsbeschränkungen für Erwachsene in Heimen. In: Pflegerecht, S. 67-75. https://pflegerecht.recht.ch/de/ artikel /01pf0218wis/freiheitsbeschrankungen-fur-erwachsene-heimen [23.01.2020].

NICE National Institute für Health and Care Excellence (2015). Guideline. Challenging behaviour and learning disabilities. Prevention and interventions for people with learning disabilities whose behaviour challenges. https://www.nice.org.uk/ guidance/ng11 [23.01.2020].

NKVF Nationale Kommission zur Verhütung von Folter (2016). Tätigkeitsbericht. Freiheitsbeschränkende Massnahmen in psychiatrischen Einrichtungen. https://www.humanrights.ch/de/menschenrechte-schweiz/inneres/strafen/ freiheitsentzug/freiheitsbeschraenkende-massnahmen-psychiatrische-einrichtungen [23.01.2020].

Ogg-Groenendaal, Marloes; Hermans, Heidi; Claessens, Brigitte (2014). A systematic review on the effect of exercise interventions on challenging behavior for people with intellectual disabilities. In: Research in Developmental Disabilities, 35(7), S. 1507-1517.

Olivier-Pijpers, Vanessa C.; Cramm, Jane M.; Buntinx, Wil H.E.; Nieboer, Anna P. (2018). Organisational environment and challenging behaviour in services for people with intellectual disabilities: A review of the literature. ALTER, Euro- pean Journal of Disability Research 12 (2019): 238-253.

Palmowski, Winfried (2015). Nichts ist ohne Kontext. Systemische Pädagogik bei « Verhaltensauffälligkeiten». Dortmund: verlag modernes lernen.

Schorro, Ewald (2015). Freiheitsbeschränkende Massnahmen in Pflegeheimen in der Schweiz. Bestimmung der Prävalenz und assoziierter organisationsbezogener Merkmale. Inaugural-Dissertation. Universität Witten/Herdecke.

Theunissen, Georg (2016). Geistige Behinderung und Verhaltensauffälligkeiten (6. Aufl.). Bad Heilbrunn: Verlag Julius Klinkhardt.

Theunissen, Georg (2019a). Autismus und herausforderndes Verhalten. Praxisleitfaden positive Verhaltensunterstützung. Freiburg im Breisgau: Lambertus Verlag.

Theunissen, Georg (2019b). Umgang mit schwerwiegendem herausforderndem Verhalten bei Erwachsenen mit komplexen Behinderungen. Ergebnisse aus einem Forschungsprojekt in Baden-Württemberg. In: Teilhabe, 4(58), S. 154-160.

UNBRK Übereinkommen über die Rechte von Menschen mit Behinderungen https://www.admin.ch/opc/de/ classified-compilation/20122488/index. html [23.01.2020].

Wüllenweber, Ernst (2009). Krisen und Behinderung. Entwicklung einer praxisbezogenen Theorie zum Verstehen von Krisen und eines Handlungskonzeptes für die Krisenintervention bei Menschen mit geistiger Behinderung und bei Autismus (3.Aufl.). Hamburg: Elbe-Werkstätten.

ZGB Schweizerisches Zivilgesetzbuch https://www.admin.ch/opc/de/classified-compilation/19070042/index.html\#id-2-3 [23.01.2020] .

Zúñiga, Franziska; Ausserhofer, Dietmar; Serdaly, Christine; Bassal, Catherine; De Geest, Sabine; Schwendimann, René (2013). SHURP. Schlussbericht zur Befragung des Pflege- und Betreuungspersonals in Alters- und Pflegeinstitutionen der Schweiz. Basel: Universität Basel, Institut für Pflegewissenschaft. 


\section{Biografische Angaben}

Büschi, Eva: Prof. Dr., Dozentin an der Hochschule für Soziale Arbeit der Fachhochschule Nordwestschweiz, Arbeitsschwerpunkte: Herausfordernde Verhaltensweisen von Menschen mit kognitiven Beeinträchtigungen, Gewalt, Trauma, Kooperative Prozessgestaltung und Projektmanagement. Letzte Publikation: Büschi, E./Calabrese, S. (Hrsg.): Herausfordernde Verhaltensweisen in der Sozialen Arbeit. Stuttgart Kohlhammer Verlag. E-Mail: eva.bueschi@fhnw.ch

Schicka, Manuela: Dr., Wissenschaftliche Mitarbeiterin an der Hochschule für Soziale Arbeit der Fachhochschule Nordwestschweiz, Arbeitsschwerpunkte: Gerontologie, Quantitative Sozialforschung. Letzte Publikation: Calabrese, S./Hassler, B./Büschi, E./Schicka, M./ Zambrino, N. (2019): Merkmale spezialisierter Institutionen für Menschen mit kognitiven Beeinträchtigungen und herausfordernden Verhaltensweisen. VHN, 88, 1-15. E-Mail: manuela. schicka@fhnw.ch

Calabrese, Stefania: Prof. Dr., Dozentin an der Hochschule Luzern - Soziale Arbeit, Arbeitsschwerpunkte: Herausfordernde Verhaltensweisen von Menschen mit kognitiven Beeinträchtigungen, Lebensqualität und Bildung im Kontext von Behinderung, agogische Aspekte bei schwerer Behinderung. Letzte Publikation: Calabrese, S./Hassler, B./Büschi, E./Schicka, M./Zambrino, N. (2019): Merkmale spezialisierter Institutionen für Menschen mit kognitiven Beeinträchtigungen und herausfordernden Verhaltensweisen. VHN, 88, 1-15.

Hassler, Benedikt: MA, Wissenschaftlicher Mitarbeiter an der Hochschule für Soziale Arbeit der Fachhochschule Nordwestschweiz, Arbeitsschwerpunkte: Arbeitsintegration, Sozialpolitik. Letzte Publikation: Calabrese, S./Hassler, B./Büschi, E./Schicka, M./Zambrino, N. (2019): Merkmale spezialisierter Institutionen für Menschen mit kognitiven Beeinträchtigungen und herausfordernden Verhaltensweisen. VHN, 88, 1-15. E-Mail: benedikt.hassler@fhnw.ch Zambrino, Natalie: MA, Wissenschaftliche Mitarbeiterin an der Hochschule Luzern - Soziale Arbeit, Arbeitsschwerpunkte: Angehörige von Menschen mit Beeinträchtigungen, Herausfordernde Verhaltensweisen von Menschen mit kognitiven Beeinträchtigungen. Letzte Publikation: Calabrese, S./Hassler, B./ Büschi, E./Schicka, M./Zambrino, N. (2019): Merkmale spezialisierter Institutionen für Menschen mit kognitiven Beeinträchtigungen und herausfordernden Verhaltensweisen. VHN, 88, 1-15. E-Mail: natalie.zambrino@hslu.ch 